should be told of the decision and the child's general practitioner and health visitor informed in writing.

We thank the health visitors who collected the data for this study. UW received a grant from the Northern Regional Health Authority.

1 Wairyar U, Richmond S, Hey E. Pregnancy outcome at 24-31 weeks gestationneonatal survivors. Arch Dis Child 1989;64:678-86.
2 Vohr BR, Oh W. Age of diphtheria, tetanus and pertussis immunisations of special care nursery graduates. Pediatrics 1986;77:569-71.

3 American Academy of Pediatrics. Report of the committee on infectious diseases (the red book). 19th ed. Illinois: American Academy of Pediatrics, 1982.

4 Lingam SL, Pateman J, Beaver R, et al. Immunisation of preterm infants. Br Med F 1986;292:1183-4.

5 Department of Health and Social Security. Immunisation against infectious disease. London: HMSO, 1988:4

(Accepted 17 fuly 1989)

\title{
Use of cyanoacrylate tissue adhesive for closing facial lacerations in children
}

\section{David P Watson}

\section{Guy's Hospital, London SE1 9RT \\ David P Watson, FRCSI, registrar in accident and emergency medicine and plastic surgery}

BrMed f 1989;299:1014
I report a prospective trial of cyanoacrylate (Histoacryl) tissue adhesive in closing facial lacerations in children aged under 14. A new method of applying the glue by means of capillary tubing was also assessed.

\section{Materials, methods, and results}

Any facial laceration requiring suturing was deemed suitable for gluing except $(a)$ lacerations longer than $3 \mathrm{~cm},(b)$ those including eyelids or the mucocutaneous junction of the mouth, $(c)$ those with ragged edges, and (d) deep or haemorrhagic wounds. Glue was applied by means of glass capillary tubing $7.5 \mathrm{~cm}$ long and $1.5 \mathrm{~mm}$ diameter. The tip of the phial containing the glue was cut at its lowest point to accommodate the applicator. The glue moves up the tubing by capillary attraction.

The skin edges were held together and the glue dabbed along the wound as a thin film. The wound was held for 30 seconds or until the glue became opaque, signifying polymerisation. Dressings were not applied unless the child was very young, when a plaster was used. Parents were advised to keep the area dry for 48 hours and then treat as normal.

Fifty children with lacerations were glued and photographed. Forty five patients returned at two weeks for review, 40 at three months, and 21 at six months. At three months cosmetic results were so good
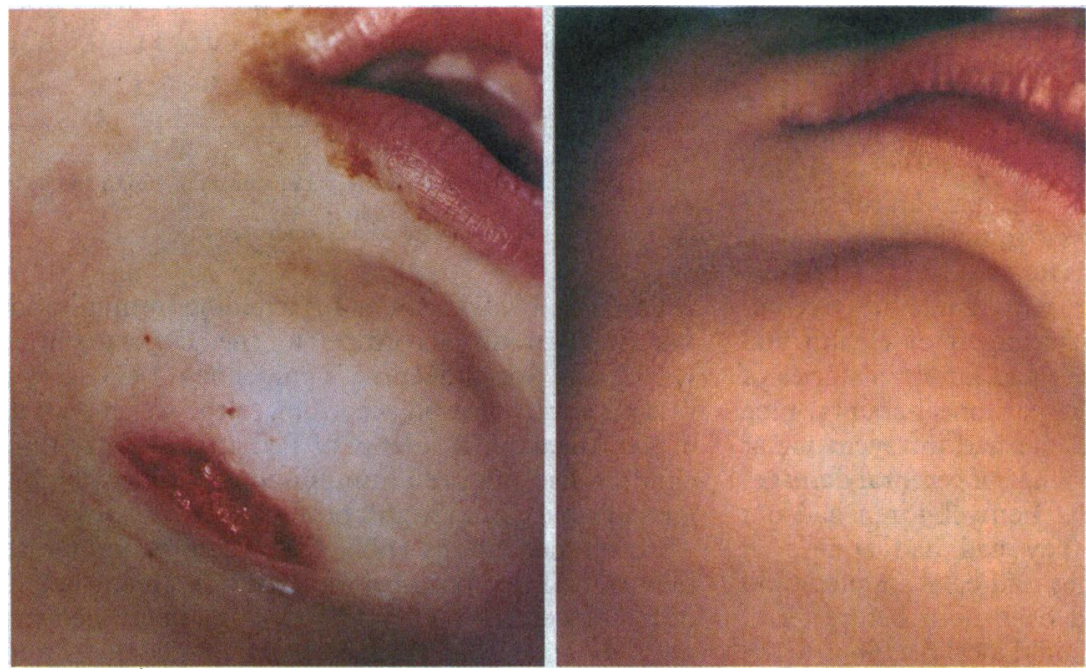

that many patients were not asked to return at six months (figure). Of the $\mathbf{4 0}$ patients reviewed at three months, 35 had wounds that had healed satisfactorily with good cosmetic results, and five had complications (detailed below). There were no problems associated with using the capillary tubing. Staff found it easy to apply and control the amount of glue used, and the time taken for gluing was considerably less than that for suturing (average five minutes as compared with 15). Consequently the procedure was far more acceptable to patients, parents, and medical staff alike.

Complications occurred in five patients. Two wounds became infected, and both were on the forehead. One was cleaned and sutured and healed with a good cosmetic result at three months. A Steristrip was applied to the other, but at three months the scar had spread. In one 7 year old a deep cheek laceration which crossed the nasolabial fold healed well but at three months showed hypertrophic scarring. In another case a 2 year old picked and reopened the wound, and at the parents' request the wound was sutured. Finally, one scar was "unusual." A 10 year old had a laceration on his chin glued. The scar faded but developed pearl white growths along its length. Histologically the scar showed keratin pits consistent with an inclusion body type reaction.

\section{Comment}

Gluing is quick, atraumatic to all concerned, and cost effective,' particularly when capillary tubing is used. Furthermore, the cosmetic results are excellent. Some children experience minor discomfort from the heat of the polymerisation, but this is less painful than injections. The cost per application is $45 \mathrm{p}$ compared with $90 \mathrm{p}$ for a $5 / 0$ nylon suture.

It is undesirable to use the same phial on different patients, even with trimming the tip of the phial. The use of capillary tubing overcomes this problem. Cultures of glue taken at weekly intervals were negative.

The wound is assessed by a casualty officer, and, if suitable, the senior nursing staff in our paediatric accident and emergency department now perform the gluing routinely. The child may be discharged with instructions to return only if there are complications; reattendance is not mandatory. This contrasts with suturing, after which the patient must have the sutures removed.

I am grateful to Dr L Stimmler and Major General N G Kirby for generous advice and to the staff of the paediatric accident and emergency department at Guy's Hospital.

1 Dalui A, Faria M, Pierto A. Non-suture closure of wounds using cyanoacrylate. f Postgrad Med 1986;32:97-100.

(Accepted 26 July 1989) 